\title{
Patient satisfaction with out-of-hours dental services
}

\author{
The effectiveness of out-of-hours dental services: Il. patient satisfaction
}

\section{R. Anderson, D. W. Thomas and C. J. Phillips Br Dent J 2005; 198: 151-156}

\section{Objective}

To compare patients' satisfaction with four types of out-of-hours emergency dental service, including both 'walk-in' and telephone-access services.

\section{Basic design}

Postal questionnaire survey of patients who had attended weekend emergency dental services. Patient satisfaction measured using an adapted version of a questionnaire developed for assessing out-of-hours medical services.

\section{Setting and subjects}

Two health authorities in South Wales, UK. The 411 patients who saw a dentist and completed the patient satisfaction questionnaire.

Results

The quality of the dentist-patient encounter was similar across services, with most patients being satisfied with the dentist's attitude and manner, the explanations and advice given, and having to see an unfamiliar dentist. Satisfaction was lower, and differed more across services in relation to service accessibility and delays in getting to see a dentist out-of-hours. The walk-in services were perceived as the least accessible: around 40\% said they had problems contacting a dentist when the surgery was closed (compared with $16 \%$ and 29\% in the other two, telephone-access services). Only $12-14 \%$ of telephone-access patients said they would be 'happy with advice plus a reliable appointment when surgeries re-opened', whereas almost half of walk-in patients thought this.

\section{Conclusions}

Despite overall satisfaction with the dentist-patient encounter, there was relative dissatisfaction with the accessibility of all services, especially the walk-in services. Out-of-hours dental services should be better designed to reflect patients' needs: the need for telephone advice as well as face-to-face consultations, and greater awareness that theoretically available services may be difficult to access unless public expectations and awareness are raised.

\section{IN BRIEF}

- This is the first comparative study of patient satisfaction with different out-of-hours dental services.

- There was overall service satisfaction, particularly with the dentist-patient encounter, but walk-in out-of-hours services were perceived as harder to access than those based on telephone access.

- Walk-in emergency treatment sessions may also be costinefficient, since almost half of attending patients felt they would have been 'happy with advice plus a reliable appointment when surgeries re-opened'.

- Some key questions are suggested that might be included in a shorter and more meaningful satisfaction instrument for out-ofhours dental patients.

- For better service design, future research should try to explan

\section{COMMENT}

This paper is the second in a series on the effectiveness of out-ofhours dental services and attempts to compare patient satisfaction across four different services types of out-of-hours service provision in South Wales. The survey provides a very useful pilot study and provides many pointers that others may build on and develop.

The results show that most patients attending all four services expressed overall satisfaction, and very few expressed dissatisfaction. However there were differences in the various elements of satisfaction and this may have been related to factors other than the actual service type provided. Such factors as the triage arrangements adopted by the call handling services, longer waiting times, differences in distance travelled to access services, case mix and whether the patients were registered or not would have had an impact on the level of satisfaction. The important finding in this study is that walk-in services were - paradoxically - perceived to be much harder to access than services initially contacted by telephone. Whilst this difference may be due to these services being poorly publicised it may also reflect dissatisfaction with having to visit even just for advice. Almost half of walk-in patients reported that they would have been happy with advice plus a reliable appointment when surgeries reopened.

NHS Direct (NHSD) has been providing this service in many areas. Its aim has been to provide easier and faster access to information and advice on health, illness and services so that callers are better able to care for themselves and their families. ${ }^{1}$ However more research is needed on how telephone advice and triage is best employed for emergency dental patients.

There is a need for the development of national principles and standards on which local services can be developed. In addition, more concerted efforts to raise public awareness of how and when access to care must be developed. The distinction between registered and non-registered patients is likely to disappear under new contractual arrangements and the current provision in some areas of providing separate emergency dental services for these groups is totally out of step with changes in other out-of-hours services. Working with the profession, the aim of future local services both within surgery hours and out-of-hours must be to ensure that patients are provided with appropriate and accurate advice and if necessary care by an appropriate health professional in the most appropriate location.

\section{Evans, Consultant in Dental Public Health, North Tyneside Primary Care Trust DOI: $10.1038 /$ j.bdj.4812044}

1. Donaldson L. Telephone access to health care: the role of NHS Direct. JR Coll Physicians Lond 2000; 34: 33-35. 